\title{
HIF1-positive and HIF1-negative glioblastoma cells compete in vitro but cooperate in tumor growth in vivo
}

\author{
PAOLO FIORENZO $^{1 *}$, MARIA PATRIZIA MONGIARDI ${ }^{1 *}$, DILETTA DIMITRI $^{1}$, MAURO COZZOLINO $^{1}$, \\ ALBERTO FERRI $^{2}$, NICOLA MONTANO ${ }^{3}$, GIANLUCA TREVISI ${ }^{3}$, GIULIO MAIRA ${ }^{3}$, LUCA BATTISTINI ${ }^{1}$, \\ MARIA LAURA FALCHETTI ${ }^{1}$, ANDREA LEVI ${ }^{1}$ and ROBERTO PALLINI ${ }^{3}$ \\ ${ }^{1}$ INMM-CNR and ${ }^{2}$ IN-CNR, IRCCS Fondazione Santa Lucia, Via del Fosso di Fiorano 64, 00143 Rome; \\ ${ }^{3}$ Institute of Neurosurgery, Catholic University School of Medicine, Largo F. Vito 1, 00168 Rome, Italy
}

Received September 14, 2009; Accepted November 9, 2009

DOI: 10.3892/ijo_00000554

\begin{abstract}
Glioblastoma multiforme (GBM) is characterized by extensive angiogenesis that is mostly orchestrated by the hypoxia inducible factor HIF-1. Deregulation of HIF-1 is believed to contribute to cancer initiation and progression. However, instances have been described in which loss of HIF-1 leads to more aggressive tumors. Here we investigated the consequences of downregulating HIF-1 function in the human GBM cell line TB10, both on cell proliferation in vitro and on tumor growth in vivo. RNA interference targeting the $\mathrm{O}_{2}$-regulated HIF- $1 \alpha$ subunit efficiently reduced HIF- $1 \alpha$ expression and transcriptional induction of HIF-1-responsive genes without affecting cell growth. Thus, singularly grown wild-type and HIF- $1 \alpha$-inhibited GBM cell populations did not significantly differ in proliferation rate. However, when the two populations were co-cultured, wild-type cells overgrew the HIF- $1 \alpha$-inhibited cells. Subcutaneous grafting in nude mice of wild-type and HIF- $1 \alpha$-inhibited GBM cells lead to comparable tumor formation and growth. Interestingly, cografting of wt and HIF- $1 \alpha$ - inhibited GBM cells in nude mice resulted in more aggressive tumors, both in terms of tumor appearance and tumor growth. This suggests that cellular populations that differ in their ability to mount a response to hypoxia may compete in vitro but cooperate in vivo resulting in increased tumor aggressiveness.
\end{abstract}

\section{Introduction}

Following the initial observation by Warburg (1) it is now a well established concept that many tumor cells display a

Correspondence to: Dr Andrea Levi, INMM-CNR, IRCCS Fondazione Santa Lucia, Via del Fosso di Fiorano 64, 00143 Rome, Italy

E-mail: andrea.levi@inmm.cnr.it

*Contributed equally

Key words: HIF1, glioblastoma multiforme, tumor xenograft, cooperation 'hypoxic' phenotype even when cultured at high oxygen tension $\left(21 \% \mathrm{O}_{2}\right)(2)$. Organism and cellular adaptation to hypoxia is, to a large extent, orchestrated by hypoxia inducible factors 1 and 2 (HIF-1 and HIF-2), heterodimeric transcription factors that regulate the expression of about one hundred genes containing hypoxia responsive elements (HREs) within their regulatory regions. HIF-1 and HIF-2 targets play essential roles in processes like glycolysis, erythropoiesis and angiogenesis. HIFs are made of a constitutively expressed HIF-1ß/ARNT subunit and of $\mathrm{O}_{2}$-regulated HIF- $1 \alpha$ and HIF- $2 \alpha$ subunits. In normal cells, at high oxygen tension, the expression of the $\alpha$ subunits is downregulated mainly through posttranslational mechanisms (3). In brief, hydroxylation at specific proline residues (Pro 402 and Pro 564 in human HIF-1 $\alpha$ sequence) by $\mathrm{O}_{2}$ dependent prolyl-hydroxylases (PHDs), create the recognition motif for ubiquitin ligase complexes containing the von Hippel-Lindau (VHL) protein. The consequent, rapid proteosomal degradation of the polyubiquitinated $\alpha$ subunits ensures that HIFs activities are maintained at a low level. When the concentration of $\mathrm{O}_{2}$ is reduced, the enzymatic activity of PHDs decreases and consequently HIF- $1 \alpha$ and HIF-2 $\alpha$ escape degradation. In many tumors, HIF-1 and HIF-2 activities are deregulated being inappropriately high even in normoxia. This may be achieved through different mechanisms like the impairment of the above described degradation pathway or via overexpression of HIF- $1 \alpha$ and HIF- $2 \alpha$ mRNAs. It is widely assumed that the metabolic reprogramming that follows HIF upregulation favors tumor cell growth (4). Deregulation of HIF signaling, however, may have a cost in terms of fitness, because of increased apoptosis (5) and examples have been described in which HIF behave as a tumor suppressor (6). For instance, siRNA-mediated downregulation of HIF-2 $\alpha$ expression reduces apoptosis of human GBM cells in hypoxic conditions, and loss of function of both HIF- $1 \alpha$ and HIF- $2 \alpha$ reduces angiogenesis but promotes tumor growth in subcutaneous xenografts (6). Tumor heterogeneity is widely recognized as a mechanism that promotes cancer cell survival in face of organism defenses and anticancer therapy, because it increases tumor robustness (7). Glioblastoma multiforme (GBM), the most lethal astrocytic brain tumor, displays high level of heterogeneity. GBMs are characterized by a strong angiogenic phenotype where the aberrant vascular structures 
are unable to sustain tumor growth resulting in hypoxic regions. The hypoxic areas are necrotic and their extension often correlates with tumor aggressiveness. Hypercellular zones called pseudopalisades, which typically surround necrotic foci, show intense nuclear staining with anti-HIF- $1 \alpha$ antibodies, while adjacent cells are not stained (8). These differences may simply reflect the independent growth of clonal variants, but could also be the basis for a cooperation among tumor cells that increases cancer aggressiveness. In this study we show that human GBM cells with reduced HIF-1 $\alpha$ expression have similar tumorigenic potential as wild-type cells when subcutaneously grafted in nude mice. Unexpectedly, cografting HIF-1 $\alpha$ deficient and wild-type cells produces more aggressive tumors than grafting single cell populations.

\section{Materials and methods}

Cell cultures and generation of stably transduced cell lines. $293 \mathrm{~T}$ cells and TB10, a cell line established in our laboratory from a secondary human GBM tumor (9) were grown in DMEM (high glucose, Invitrogen Italia, Milan, Italy) supplemented with $10 \%$ heat-inactivated fetal bovine serum. For experiment requiring hypoxic conditions the cells were placed in a modular incubator chamber flushed with a gas mixture containing $2 \% \mathrm{O}_{2}, 5 \% \mathrm{CO}_{2}, 93 \% \mathrm{~N}_{2}$ at $37^{\circ} \mathrm{C}$. Silencing of HIF-1 $\alpha$ was obtained by retroviral mediated expression of shRNA targeting nucleotide 528-547 of HIF-1 $\alpha$ sequence (GenBank $^{\mathrm{TM}}$ accession no. NM_001530) using pRETRO-Super vector (10). Control shRNA had the following sequence: 5'-GGGATATCCCTCTAGATTA-3'. Neither the HIF-1 $\alpha$ targeting shRNA nor the control sequences have any homology with other human gene as tested by BLAST (http://ncbi.nln. nih.gov/BLAST). Retroviruses were produced in 293T cells by cotransfecting pRETRO-Super together with plasmids encoding for gag-pol and VSV-G proteins. Viral supernatant was collected $48 \mathrm{~h}$ post-transfection, filtered through a $0.45-\mu \mathrm{m}$ pore size filter and added to the cells in the presence of $2 \mu \mathrm{g} / \mathrm{ml}$ polybrene. Forty-eight hours post-infection cells were selected by $1 \mu \mathrm{g} / \mathrm{ml}$ of puromycin. TB10 expressing green or red fluorescent proteins were similarly obtained by infection with recombinant retrovirus prepared from pLEGFP-C1 and pRetroQ-DsRed Monomer C1, respectively (Clontech Laboratories, Inc.). Cells were subjected to three consecutive rounds of infection and $>80 \%$ of the cells expressed the fluorescent protein.

Cell proliferation. Growth rate was measured by seeding $2 \times 10^{5}$ cells $/ 60 \mathrm{~mm}$ dishes in triplicates, and counting cell number after $48 \mathrm{~h}$. Convenient dilution was used for successive rounds of seeding. Cell proliferation was also measured with the CellTiter $96^{\circledast} \mathrm{AQ}_{\text {ueous }}$ One Solution Cell Proliferation Assay (Promega Corp.), according to the manufacturer's instructions. To measure the relative proliferation index of cTB10 and siHIF-TB10 cells in co-culture, $10^{5}$ GFP-tagged cTB10 cells and $10^{5}$ DsRed-tagged siHif-TB10 cells were seeded per $60 \mathrm{~mm}$ dish, every $72 \mathrm{~h}$ the cells were collected and one fourth of the population was re-seeded and the remaining were used for DNA preparation. Genomic DNA was digested with the restriction enzyme EcoRI and $10 \mu \mathrm{g}$ used for quantification by real-time PCR with probes specific for GFP, DsRed and a hTERT DNA sequence in an introne region. The relative proliferation of cTB10 and siHIF-TB10 cells was calculated by comparing the RQs of GFP and DsRed with respect to hTERT in DNA preparations from cells at different passages of co-culture to the RQs in a DNA preparation from equal number of cTB10 and siHIF-TB10 cells.

Real-time RT-PCR. Total RNA $(2 \mu \mathrm{g})$ was retrotranscribed according to standard procedures and one tenth of the cDNA was used to quantify the transcripts by real-time RT-PCR using probes from the Universal ProbeLibrary (Roche Applied Science) and gene specific-primers designed by the Probe Finder software (Roche Applied Science). RQ were calculated relative to TBP mRNA assumed to be constant. The real-time PCR was performed with the 7900HT Fast Real-Time PCR System by Applied Biosystem.

Western blotting. Approximately $10^{6}$ cells were lysed in $10 \mathrm{mM}$ HEPES pH 8.0, $50 \mathrm{mM} \mathrm{NaCl}, 500 \mathrm{mM}$ sucrose, $0.1 \mathrm{mM}$ EDTA, $0.5 \%$ Nonidet P- $40,5 \mathrm{mM} \mathrm{MgC}_{12}$, containing protease inhibitors (Sigma). Nuclear pellets were washed twice with the same buffer and nuclear proteins were extracted by high salt buffer (20 mM HEPES pH 7.9, $420 \mathrm{mM} \mathrm{NaCl}, 25 \%$ glycerol, $0.2 \mathrm{mM}$ EDTA, $1.5 \mathrm{mM} \mathrm{MgCl}$, containing protease inhibitors (Sigma). Immunoreactive bands were visualized by the Pierce Dura Signal ECL system. HIF-1 $\alpha$ was detected with the monoclonal antibody 610958 from BD Transduction Laboratory. The anti-lamin B polyclonal antibody sc-6217 by Santa Cruz Biothecnology Inc. was used to assess equal loading of nuclear proteins.

Glioblastoma cell xenografting in immunodeficient mice. The experiments on animals were approved by the Ethics Committee of the Catholic University School of Medicine, Rome, Italy. Four-week-old male nude mice (Harlan, Udine, Italy) were used as hosts for the in vivo models of tumorigenesis. TB10 cells were harvested, washed twice, and resuspended in cold PBS at the concentration of $1 \times 10^{4} / \mu 1$. Then, $100 \mu 1$ of cells were mixed with $100 \mu 1$ of Matrigel (BD Bioscience, Bedford, MA) on ice, and the mixture was implanted by subcutaneous injection. Two injections were performed on a single mouse. The animals were kept under pathogen-free conditions in positive-pressure cabinets (Tecniplast Gazzada, Varese, Italy).

Histology, immunohistochemistry, and fluorescence microscopy. The Matrigel implants were removed 1 to 8 weeks after grafting. The mice were deeply anesthetized and transcardially perfused with $0.1 \mathrm{M}$ PBS (pH 7.4), followed by $4 \%$ paraformaldehyde in $0.1 \mathrm{M}$ PBS. The implants were surgically removed, stored in $30 \%$ sucrose buffer overnight at $4^{\circ} \mathrm{C}$, and either embedded in paraffin or cryotomed. Paraffinized sections (5 $\mu \mathrm{m}$ thick), were stained with H\&E for morphological analysis. The material was studied under light field illumination and images were captured with a Leitz microscope equipped with a Nikon Coolpix 995 camera connected to a PC. For fluorescence microscopy, cryotomed sections (20 $\mu \mathrm{m}$ thick) were collected in distilled water, mounted on slides, and cover-slipped with Eukitt. Images were obtained with a Laser Scanning Confocal Microscope (IX81, Olympus Inc., 
A

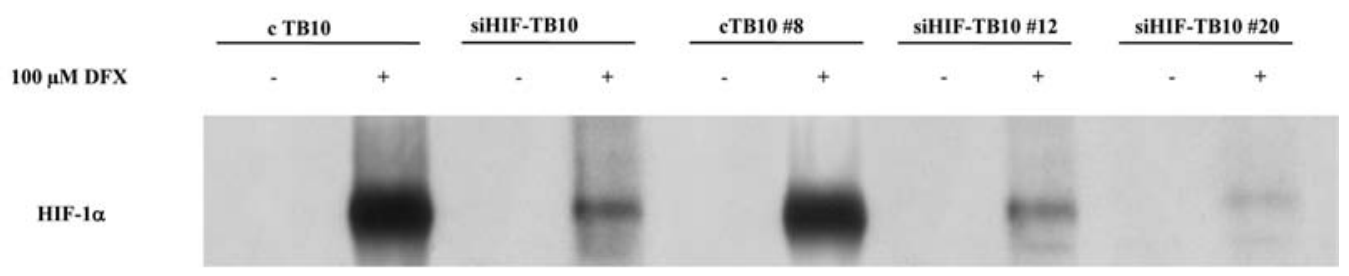

Lamin B

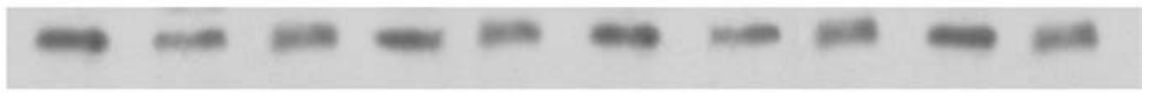

B

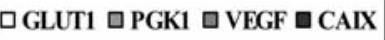

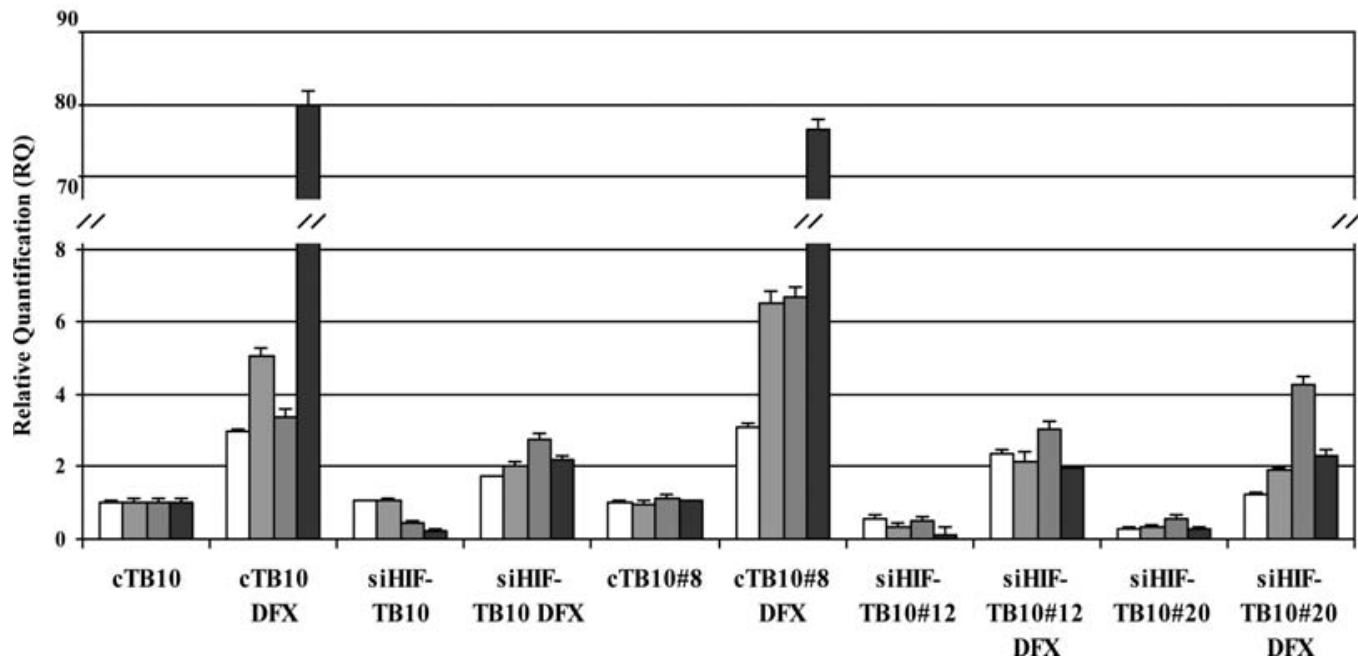

Figure 1. Impairment of HIF1 expression and activity in siHIF-TB10 cells. (A) HIF-1 $\alpha$ protein levels were measured by Western blot analysis of nuclear extracts of cells cultured for $12 \mathrm{~h}$ in the absence or in the presence of the hypoxia-mimetic deferoxamine, using the monoclonal antibody 610958 from $\mathrm{BD}$ Transduction Laboratory. The anti-lamin B polyclonal antibody sc-6217 by Santa Cruz Biothecnology Inc. was used to assess equal loading of nuclear proteins. (B) Expression of hypoxia-regulated transcripts was measured by real-time RT-PCR on total RNA extracted from cells cultured for $12 \mathrm{~h}$ in the absence or in the presence of $100 \mu \mathrm{M}$ deferoxamine. Real-time RT-PCR was performed using probes from the Universal Probe Library (Roche Applied Science) and gene specific-primers designed by the Probe Finder software (Roche Applied Science). For each gene examined the amount present in cTB10 cells grown in the absence of deferoxamine was set equal to 1 .

Melville, NY). Representative images from each slide were acquired using the confocal microscope with fixed optical parameters, light intensity, filter settings, and magnification. Acquired images were stored as TIFF files and evaluated using Adobe Photoshop 6.

Statistical analyses. Results are expressed as the mean \pm standard deviation (SD). The significance of differences was assessed by the unpaired two-tail Student-t test. Significance was set at $\mathrm{p}<0.05$.

\section{Results}

Production of GBM clones with reduced HIF-1a expression. To silence HIF-1 $\alpha$ in TB10 cells, a cell line established in our laboratory from a human GBM (9), we delivered shRNA by the pRETRO-Super retroviral vector (10). Infected cells were selected by puromycin and the resulting polyclonal population was named siHIF-TB10. Control populations were obtained by infecting TB10 cells with empty pRETROSuper (cTB 10) or with a pRETRO-Super encoding for a shRNA of random sequence that has no homology with any human transcript (siRandom-TB10). Since in every condition used, and for every parameter measured, siRandom-TB10 and cTB10 cells were indistinguishable, only the data obtained with cTB10 are shown. In addition, we isolated from the two polyclonal populations a number of independent clones. Single clones isolated from the cTB10 population had comparable expression of HIF- $1 \alpha$ mRNA as measured by real-time RT-PCR $(<10 \%$ difference within distinct clones). Those derived from siHIF-TB10 cells displayed different degrees of inhibition of HIF-1 $\alpha$, ranging from 40 to $90 \%$. For the experiments described below we used a single clone of cTB10, i.e., cTB10\#8, and two independent clones, siHIF-TB10\#12 and siHIF-TB10\#20, which showed the strongest inhibition of HIF-1 $\alpha$ expression (not shown). HIF-1 $\alpha$ protein expression was 
examined by Western blotting, using anti-HIF-1 $\alpha$ antibodies. Nuclear proteins were extracted also from cells treated overnight with $100 \mu \mathrm{M}$ deferoxamine, a hypoxia-mimetic drug that inhibits PHDs. As shown in Fig. 1A, deferoxamine treatment of cTB10 cells resulted in the induction of HIF-1 $\alpha$ protein from an undetectable band (lanes 1 and 5) to a strong signal (lanes 2 and 6). Conversely, siHIF-TB10 cells showed only a slight increase in HIF-1 $\alpha$ protein content which was even slighter in the selected single clones (lanes 4, 8 and 10). Finally, we measured to which extent the transcription of HIF-1-regulated genes was affected by HIF-1 $\alpha$ silencing. RNA was extracted from cTB10 and siHIF-TB10 cells cultured in the absence or in the presence of deferoxamine and the level of CA9, VEGF, PGK1 and GLUT1 mRNAs was measured by real-time RT-PCR. As shown in Fig. 1B, deferoxamine treatment induced, although to a different extent, the transcription of all these genes in wild-type cells. Such a transcriptional response was blunted in siHIF-TB10 cells. Of note, even in normoxia, the amount of CA9 and VEGF transcripts was significantly lower in siHIF-TB10 cells with respect to cTB10 cells.

HIF-1a suppression does not alter cell proliferation but cTB10 cells out compete siHIF-TB10 cells in mixed culture. It was recently shown that cells with reduced HIF- $1 \alpha$ expression grow faster than wild-type cells when cultured in normoxic conditions in the presence of growth factors (11). To assess if proliferation of TB10 cells was similarly altered by HIF- $1 \alpha$ downregulation, we measured the growth rate of cTB10 pool, cTB10\#8, siHIF-TB10 pool, siHIF-TB10\#12 and siHIF-TB10\#20 cells. There was no significant change in the rate of population doubling among the different TB10 cell populations (doubling time of $28.5 \pm 2.2,30.6 \pm 2.4$, $33.2 \pm 3.2,32.7 \pm 2.2$ and $30.4 \pm 2.5 \mathrm{~h}$ for cTB10 pool, cTB10\#8, siHIF-TB10 pool, siHIF-TB10\#12 and siHIF-TB10\#20 respectively). Therefore our findings differ from those by Lum and colleagues (11), which were obtained in immortalized bone marrow derived IL-3 dependent cells, in primary cultures of T cells or in NIH 3T3 cells. All these cell types are essentially untransformed and do not possess those additional alterations that contribute to the cancerous phenotype of GBM cells and that may uncouple cell growth from cell proliferation.

Although cTB10 and siHIF-TB10 cells showed similar proliferation rate in standard conditions, they still may differently utilize nutrients present in the medium and secrete diverse metabolites, in other words they may differently change the extracellular environment. We thus decided to examine if the growth rate of each cell population was affected by the presence of the other cell type. To distinguish the two cell types in mixed co-cultures, we generated cTB10\#8 cells expressing the enhanced green fluorescent protein (EGFP) and siHIF-TB10\#12 cells expressing the red fluorescent protein (DsRed). In a preliminary experiment, we showed that the expression of the fluorescent proteins did not modify the proliferation rate of the cells (not shown). We then mixed the two populations at 1:1 ratio and grew them in normoxia or in hypoxic conditions. The co-culture was split 1:4 every three days and at day $0,3,9$, and 15 genomic DNA was extracted. The relative abundance of cTB10\#8 and of siHIF-TB10\#12 cells was measured by quantifying, through
A

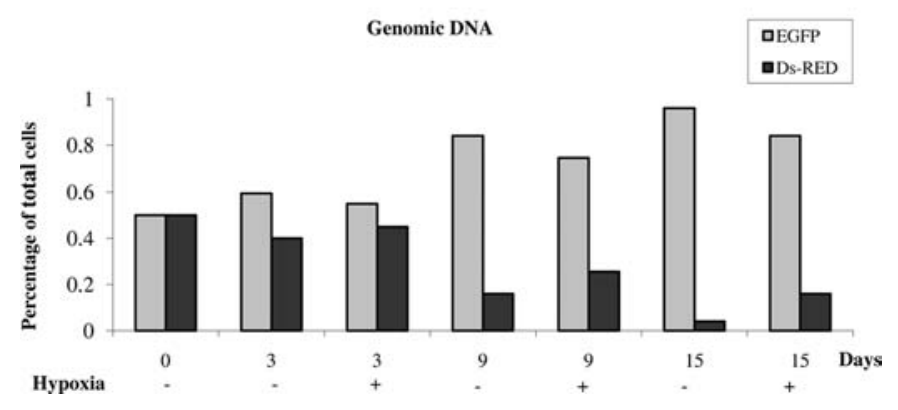

B

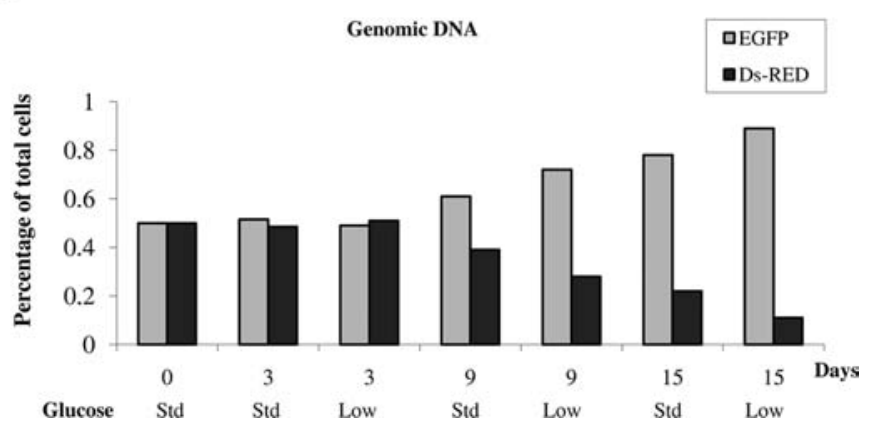

Figure 2. Relative growth of control and siHIF-TB10 cells in co-colture. (A) The relative number of cTB10\#8 expressing EGFP and siHIF-TB10\#12 expressing DsRed was assessed by measuring the ratio of EGFP and DsRed-encoding DNA as measured by real-time PCR. The values are expressed setting the ratio at time 0 equal to 0.5 . Cells were grown in normoxia or in hypoxia. (B) Cells were grown in normoxia in standard $(25 \mathrm{mM})$ or reduced $(5 \mathrm{mM})$ glucose concentration.

real-time PCR, the amount of DNA encoding for EGFP and DsRed protein respectively. The data were normalized using an exon of the telomerase catalytic subunit hTERT as an internal standard. As shown in Fig. 2A, cTB10 cells increased in relative abundance with respect to siHIF-TB10 cells in long-term co-cultures. Hypoxia only slowed down, without preventing the outgrowth of cTB10 relative to siHIF-TB 10 cells. A likely explanation for the reduced duplication rate of siHIF-TB10 with respect to cTB10 in cocolture is that some components in the medium may become limiting. Tumor cells require high flux of glucose for fast synthesis of ATP through the glycolytic pathway and for production of anabolites necessary for cell growth and cell duplication (4). HIF-1 is a major positive regulator of glucose uptake and aerobic glycolysis. We thus reasoned that siHIFTB10 cells, which likely consume glucose at a lower rate than cTB10 cells, may be outcompeted because of sub-optimal concentration of glucose. If this is the case, the difference in growth between co-cultured cTB10 and si HIF-TB10 cells should be exacerbated in low glucose medium. As shown in Fig. 2B, a 5-fold reduction of glucose concentration from 25 to $5 \mathrm{mM}$ caused a more rapid outgrowth of cTB 10 cells, demonstrating that the siHIF-TB10 cells are much more sensitive to glucose deprivation than cTB10. 


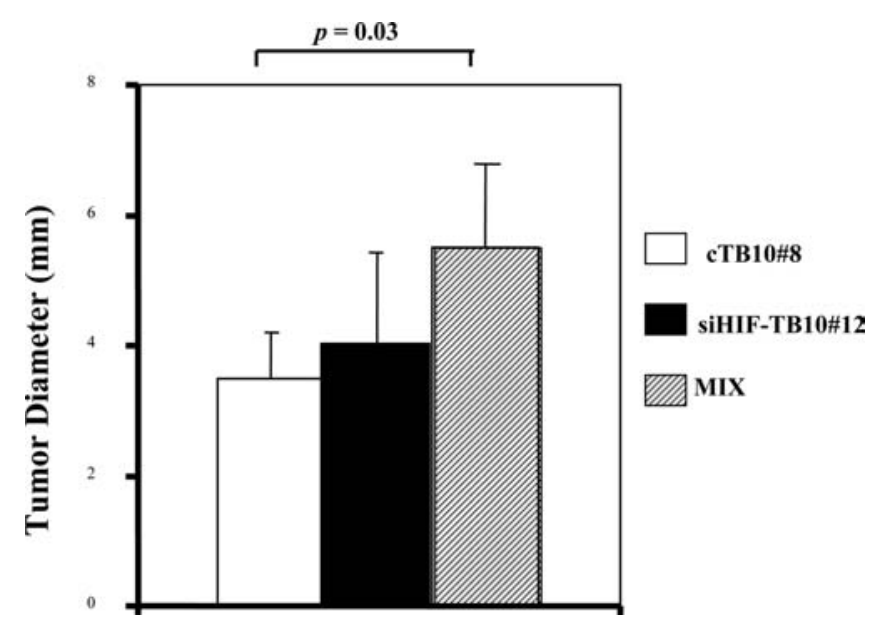

Figure 3. Tumor growth of xenografts derived from cTB10\#8 cells, siHIF-TB10\#12 cells and mixed populations. cTB10 cells, siHIF-TB10 cells, cTB10 cells plus siHIF-TB10 cells were sucutaneously injected in athymic mice as Matrigel implants. The mean tumor diameter was measured 8 weeks post grafting.

Xenografts of mixed population of wtTB10 and siHIF-TB10 cells grow faster than xenografts of either wtTB 10 or siHIF-TB10 cells. We next assessed whether the inhibition of HIF-1 signaling may affect the growth of TB10 GBM cells in vivo. Either $10^{6}$ EGFP-expressing cTB10\#8 cells or $10^{6}$ DsRed-expressing siHIF-TB10\#12 cells were subcutaneously implanted in immunocompromised nude mice. Tumor growth was monitored over an 8 -week period. To investigate whether the cTB10 and siHIF-TB10 cell populations competed in vivo as they did in vitro, a third group of animals was grafted with a 1:1 mixture of $5 \times 10^{5}$ EGFP-expressing cTB10\#8 cells and $5 \times 10^{5}$ DsRed-expressing siHIF-TB10\#12 cells. After 4 weeks, tumor take was 37\% (n, 3/8), 37\% (n, 3/8), and 75\% (n, 6/8) in the cTB10\#8 group, siHIF-TB10\#12 group, and cTB10\#8/ siHIF-TB10\#12 group, respectively. Moreover, 8 weeks after grafting, the mean diameter of the tumor xenografts was significantly greater in the cTB10\#8/siHIF-TB10\#12 grafted mice as compared both with the cTB10\#8 and with the siHIF-TB10\#12 grafted mice $(\mathrm{p}<0.05$, Student's t-test; Fig. 3). On histological examination, the xenografts showed the typical appearance of GBM without substantial differences of the tumor cytoarchitecture and vasculature among the different groups. When we analysed the mixed xenografts under fluorescent microscopy, by week 2 after grafting, we did not find major differences in the topographical distribution of the EGFP-expressing cTB10\#8 and the DsRed-expressing siHIF-TB10\#12 cells (Fig. 4A). By week 4 after grafting, however, the central region of the mixed tumor xenografts was mostly populated by DsRed-expressing siHIF-TB10\#12 (Fig. 4B). Thus, as opposed to that occurring in vitro, siHIF-TB10 GBM cells are not overgrown by cTB10 cells and even exhibit a growth advantage in the central region of the tumor.

\section{Discussion}

Conflicting results on the role of HIF-1 $\alpha$ on xenograft growth have been published. Silencing of HIF-1 $\alpha$ by RNAi was demonstrated to attenuate human glioma cell growth in xenograft models (12), to result in a transient reduction of established tumor growth or to have no effect on tumor growth, depending on the tumor cell line (13). Finally, expression of a dominant negative form of HIF- $1 \alpha$ in a rat glioma promoted tumor growth possibly by reducing apoptosis under hypoxia (6). Such heterogeneity of behavior in experimental tumors parallels clinical data: elevated HIF-1 and HIF-2 expression was generally found to correlate with poor prognosis (recently reviewed in ref. 14), but patients with HIF-1 $\alpha$-positive nonsmall cell lung carcinomas had a longer survival than HIF-1 $\alpha$ negative patients (15), and expression of HIFa in surgically treated patients with head and neck squamous cell carcinoma was associated with improved disease-free and overall survival (16).

Possibly the most relevant result of our work is that tumor xenografts derived from mixed populations of HIF- $1 \alpha$-positive and HIF-1 $\alpha$-negative GBM cells were more aggressive than those obtained by grafting each cell population. We propose two, not necessarily contradictory, explanations for the increased growth of tumors from mixed populations of HIF- $1 \alpha$-positive and HIF- $1 \alpha$-negative TB10 cells. According to the first hypothesis, mixed tumors grow faster because the two populations colonize different microenvironments. In protracted and extreme hypoxia, HIF-1 induces the synthesis of pro-apoptotic genes, thus siHIF-TB10 cells may be more resistant to hypoxia-induced apoptosis and survive in oxygenpoor niches which are not permissive for the cTB10 cells. In well vascularized regions, cTB10 and siHIF-TB10 cells may grow at a similar rate, while at a certain distance from blood vessel, where nutrients such as glucose may become limited, wild-type cells may have a growth advantage. Fluorescence microscopy examination of the tumors at later times appears to corroborate this hypothesis, At early times, however, the two populations of cTB10 and siHIF-TB10 cells appear well interspersed and homogeneously distributed within the tumor mass.

According to the second hypothesis, cTB10 and siHIFTB10 cells actually cooperate, so that each population gains a growth advantage from the presence of the other one. Koukourakis et al (17) suggested that cancer cells with an 'anaerobic metabolism' and tumor associated stroma cells, which display 'aerobic metabolism' functionally cooperate by exchanging metabolites and buffering potentially toxic products (17). Recently it was shown that tumor cells relying on glycolysis for energy production secrete large amounts of lactate that is taken up and utilized by neighboring cells more proficient in oxydative phosphorylation (18). Metabolic cooperation may as well occur between transformed cell populations like cTB10 and siHIF-TB10 that differ in their glucose utilization because of different HIF-1 activities. Cooperation among tumor cells with different genotypes has been proposed as a major driving force in tumor progression (19) and suggests that the hallmarks of cancer (20) are not necessarily cell autonomous mutations that each cell within the tumor must acquire, but may be a resource of the tumor as a whole.

Our findings suggest caution in evaluating anticancer therapies based on inhibition of HIF functions. Should a hypothetical anti-HIF drug reach only a subpopulation within 


\section{A}

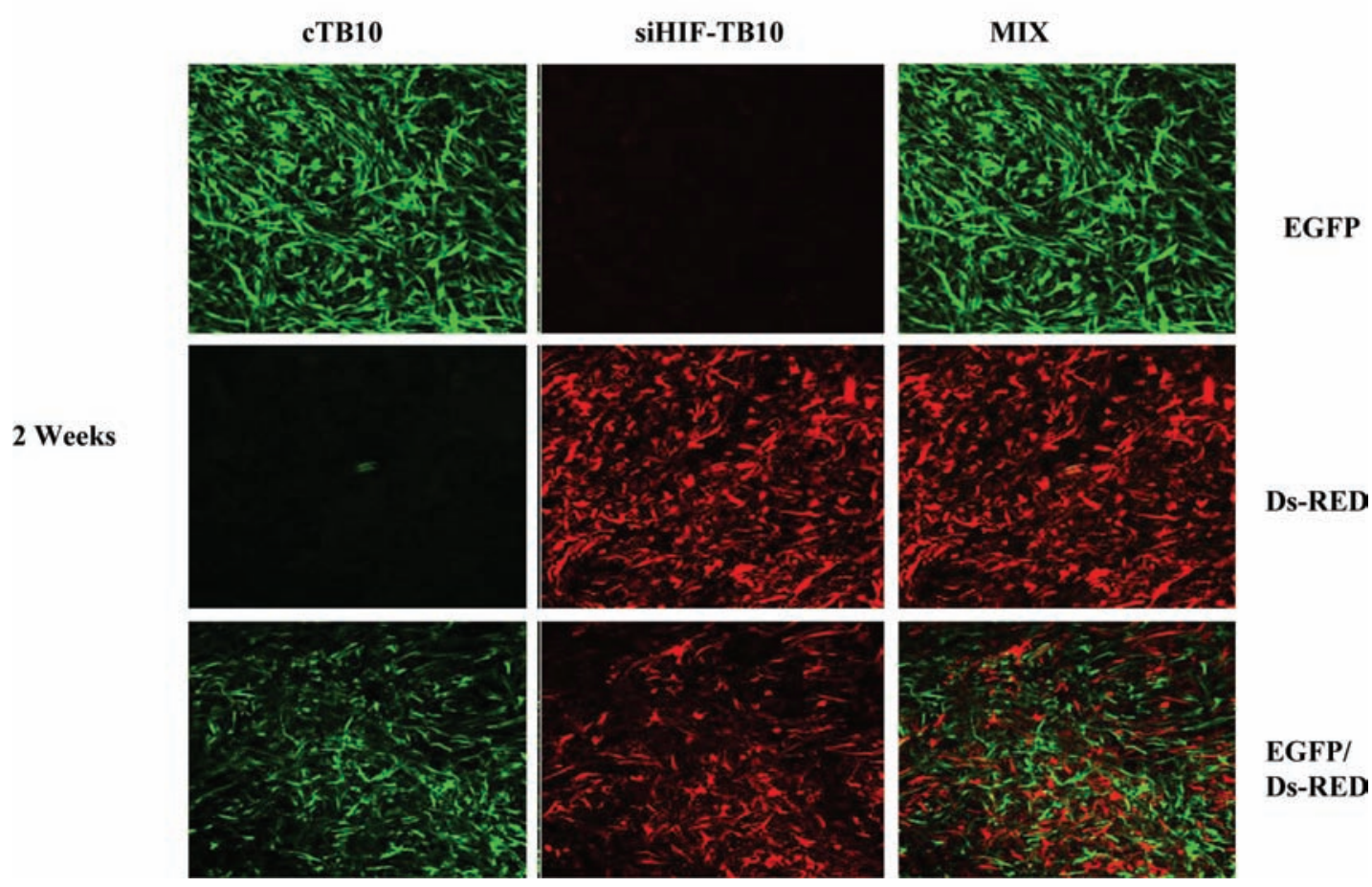

B

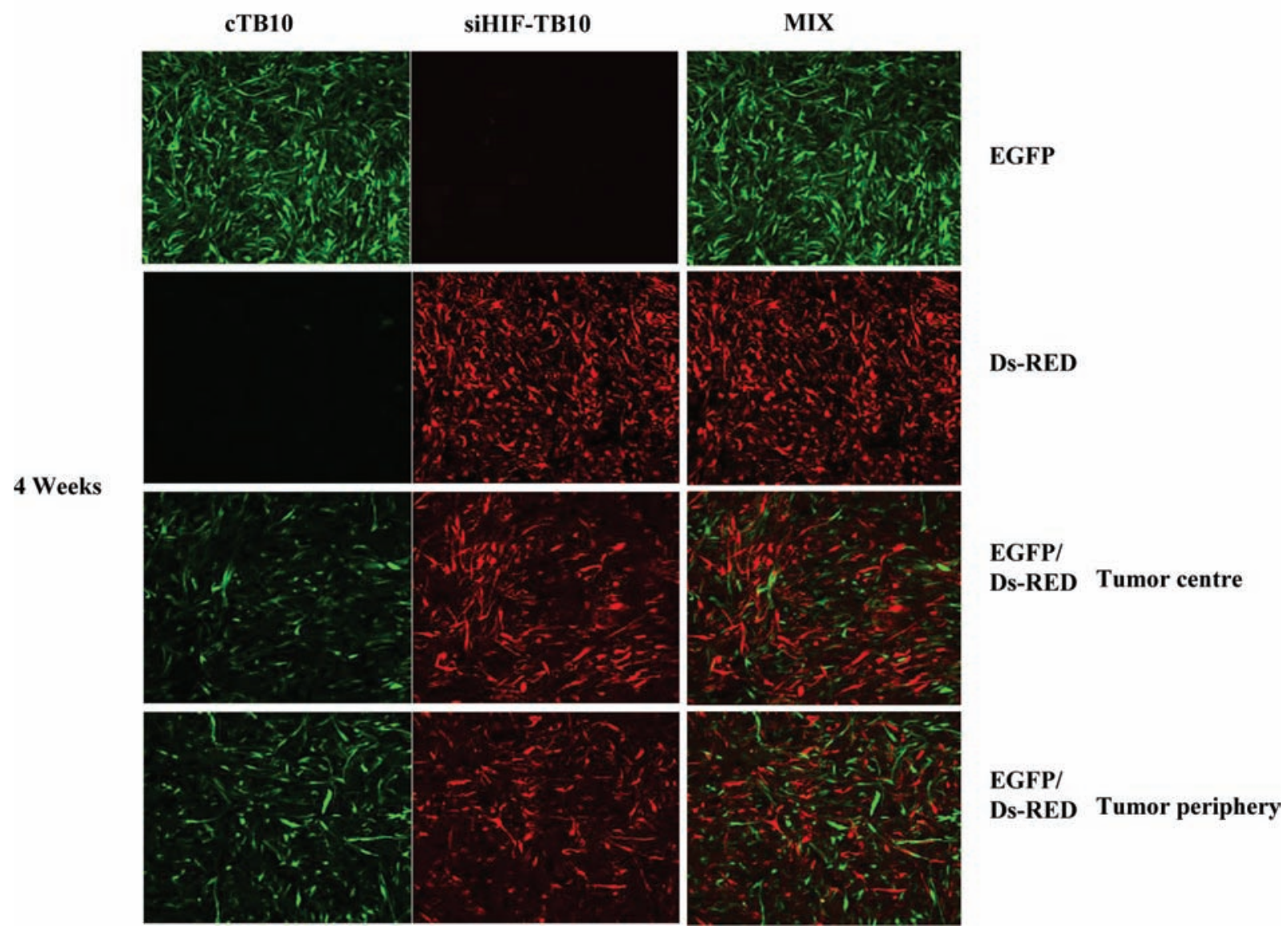

Figure 4. Distribution of cTB10\#8 and siHIF-TB10\#12 cells within the tumor. Tumors were dissected at week 2 (A) and week 8 (B) post grafting. EGFPexpressing cTB10\#8 cells and DsRed-expressing siHIF-TB10\#12 cells were detected by fluorescent microscopy (original magnification x20). 
a tumor mass, it may promote, instead of hamper, cancer growth.

\section{Acknowledgments}

This work was supported by grants from FIRB RBNE01MBEC_003, ATENA Onlus, Filas and AIRC.

\section{References}

1. Warburg O: On respiratory impairment in cancer cells. Science 124: 269-270, 1956

2. Gatenby RA and Gillies RJ: Why do cancers have high aerobic glycolysis? Nat Rev Cancer 4: 891-899, 2004.

3. Kaelin WG Jr and Ratcliffe PJ: Oxygen sensing by metazoans: the central role of the HIF hydroxylase pathway. Mol Cell 4: 393-402, 2008.

4. De Berardinis RJ, Lum JJ, Hatzivassiliou G and Thompson CB: The biology of cancer: metabolic reprogramming fuels cell growth and proliferation. Cell Metab 7: 11-20, 2008.

5. Carmeliet P, Dor Y, Herbert J M, Fukumura D, Brusselmans K, Dewerchin M, Neeman M, Bono F, Abramovitch R, Maxwell P, Koch CJ, Ratcliffe P, Moons L, Jain RK, Collen D and Keshert E: Role of HIF-1alpha in hypoxia-mediated apoptosis, cell proliferation and tumour angiogenesis. Nature 394: 485490, 1998.

6. Acker T, Diez-Juan A, Aragones J, Tjwa M, Brusselmans K, Moons L, Fukumura D, Moreno-Murciano M P, Herbert JM, Burger A, Riedel J, Elvert G, Flamme I, Maxwell PH, Collen D, Dewerchin M, Jain RK, Plate KH and Carmeliet P: Genetic evidence for a tumor suppressor role of HIF-2alpha. Cancer Cell 8: 131-141, 2005.

7. Kitano H: Cancer as a robust system: implications for anticancer therapy. Nat Rev Cancer 4: 227-235, 2004.

8. Brat DJ, Castellano-Sanchez AA, Hunter SB, Pecot M, Cohen C, Hammond EH, Devi SN, Kaur B and van Meir EG: Pseudopalisades in glioblastoma are hypoxic, express extracellular matrix proteases, and are formed by an actively migrating cell population. Cancer Res 64: 920-927, 2004.

9. De Stasio G, Casalbore P, Pallini R, Gilbert B, Sanita F, Ciotti MT, Rosi G, Festinesi A, Larocca LM, Rinelli A, Perret D, Mogk DW, Perfetti P, Mehta MP and Mercanti D: Gadolinium in human glioblastoma cells for gadolinium neutron capture therapy. Cancer Res 61: 4272-4277, 2001.
10. Brummelkamp TR, Bernards R and Agami R: Stable suppression of tumorigenicity by virus-mediated RNA interference. Cancer Cell 2: 243-247, 2002.

11. Lum JJ, Bui T, Gruber M, Gordan JD, De Berardinis RJ, Covello KL, Simon MC and Thompson CB: The transcription factor HIF-1alpha plays a critical role in the growth factordependent regulation of both aerobic and anaerobic glycolysis. Genes Dev 21: 1037-1049, 2007.

12. Gillespie DL, Whang K, Ragel BT, Flynn JR, Kelly DA and Jensen RL: Silencing of hypoxia inducible factor-1alpha by RNA interference attenuates human glioma cell growth in vivo. Clin Cancer Res 13: 2441-2448, 2007.

13. Li L, Lin X, Staver M, Shoemaker A, Semizarov D, Fesik SW and Shen Y: Evaluating hypoxia-inducible factor-1alpha as a cancer therapeutic target via inducible RNA interference in vivo. Cancer Res 65: 7249-7258, 2005.

14. Rankin EB and Giaccia AJ: The role of hypoxia-inducible factors in tumorigenesis. Cell Death Differ 15: 678-685, 2008.

15. Volm M and Koomagi R: Hypoxia-inducible factor (HIF-1) and its relationship to apoptosis and proliferation in lung cancer. Anticancer Res 20: 1527-1533, 2000.

16. Beasley NJ, Leek R, Alam M, Turley H, Cox GJ, Gatter K, Millard P, Fuggle S and Harris AL: Hypoxia-inducible factors HIF-1alpha and HIF-2alpha in head and neck cancer: relationship to tumor biology and treatment outcome in surgically resected patients. Cancer Res 62: 2493-2497, 2002.

17. Koukourakis MI, Giatromanolaki A, Harris AL and Sivridis E: Comparison of metabolic pathways between cancer cells and stromal cells in colorectal carcinomas: a metabolic survival role for tumor-associated stroma. Cancer Res 66: 632-637, 2006.

18. Sonveaux P, Vegran F, Schroeder T, Wergin MC, Verrax J, Rabbani ZN, De Saedeleer CJ, Kennedy KM, Diepart C, Jordan BF, Kelley M J, Gallez B, Wahl M L, Feron O and Dewhirst MW: Targeting lactate-fueled respiration selectively kills hypoxic tumor cells in mice. J Clin Invest 118: 3930-3942, 2008.

19. Axelrod R, Axelrod DE and Pienta KJ: Evolution of cooperation among tumor cells. Proc Natl Acad Sci USA 103: 13474-13479, 2006.

20. Hanahan D and Weinberg RA: The hallmarks of cancer. Cell 100: 57-70, 2000. 